\title{
Dinámica de los reportes corporativos presentados por las empresas multilatinas colombianas en el periodo 2016-2018 ${ }^{1}$
}

\author{
David Berrío Zapata ${ }^{2}$
}

Recibido: 03 de febrero de 2020

Aprobado: 30 de abril de 2020

Berrío, D. (2020). Dinámica de los reportes corporativos presentados por las empresas multilatinas colombianas en el periodo 2016-2018. Revista Activos, 18(1), 77-94. DoI: https://doi.org/10.15332/25005278/6158

\section{Clasificación JEL: F21, F23}

\section{Resumen}

Con el fin de reconocer la evolución de los reportes corporativos presentados por las multilatinas colombianas entre el 2016 y el 2018, se seleccionaron los informes con mayor nivel de integración de información y luego

1 Este trabajo se elaboró en el marco del proyecto de investigación "El reporte corporativo en Colombia y América Latina como mecanismo de rendición de cuentas", financiado por el Centro de Investigaciones y Consultorías y el Departamento de Ciencias Contables de la Universidad de Antioquia.

2 Estudiante de Contaduría Pública Universidad de Antioquia, octavo semestre. Correo electrónico: david.berrioz@udea.edu.co. Asesor: Jaime Andrés Correa García. Correo electrónico: jaime.correa@udea.edu.co. Profesor titular del Departamento de Ciencias Contables, Universidad de Antioquia.

ORCID: https://orcid.org/0000-0003-3473-4953 
fueron revisados bajo metodología de análisis de contenido con cinco categorías: desempeño como multilatinas, descripción del reporte, componente financiero, componente de sostenibilidad y gobierno corporativo. Como conclusión, no se puede afirmar que exista una tendencia marcada en presentación de reportes corporativos por estas empresas en dicho periodo; este no es el resultado de una única causa, aunque en Colombia se presente tendencia hacia la presentación del reporte integrado.

Palabras clave: reportes corporativos, multilatinas, reportes de sostenibilidad, reporte integrado, multinacionales.

\title{
Dynamics of Corporate Reports Submitted by Colombian Multilatina Companies during the 2016-2018 Period
}

\begin{abstract}
In order to recognize the evolution of the corporate reports presented by the Colombian multilatinas between 2016 and 2018, the reports with the highest level of information integration were selected and then reviewed using a content analysis method with five categories of analysis: performance as multilatinas, description of the report, financial component, sustainability component, and corporate governance. In conclusion, it cannot be said that there is a marked trend in the presentation of corporate reports by these companies during said period; this is not the result of a single cause, although in Colombia there is a trend towards the presentation of integrated reports.
\end{abstract}

Keywords: corporate reports, multilatinas, sustainability reports, integrated reports, multinationals. 


\section{Introducción}

Latinoamérica se convirtió en un espacio propicio para la internacionalización de las empresas, lo que las ha llevado a ser más competitivas. Esto le ha permitido evolucionar desde su estructura hasta sus procesos para mantener un lugar y mejorar su desempeño tanto en los aspectos financieros y económicos, como en los sociales y ambientales (Casanova y Dumas, 2010), los cuales se han visibilizado cada vez más pues los usuarios de la información ahora no están conformes solo con los estados financieros (AECA, 2011).

Reconociendo el proceso de globalización y fortalecimiento de las empresas de América Latina, cobraron relevancia algunas multinacionales que hoy se conocen como Multilatinas (Cuervo-Cazurra, 2007). Poniendo en comunión este conglomerado de empresas con la dinámica actual de rendición de cuentas, en la cual se opta por un deber ser que tiende a comunicar mucho más que el desempeño financiero, se pueden analizar las características de los reportes que tienden a la comunicación de contenidos integrados, emitidos por las multilatinas colombianas entre 2016 y 2018.

Los reportes sometidos a evaluación son los que se consideró que integraban más información de cada año para la empresa y que fueron publicados en sus portales de internet. Particularmente, se analizó la descripción del reporte, el componente financiero, el componente de sostenibilidad y el gobierno corporativo, todos ellos entendidos como variables que representan dimensiones materiales para la comunicación de información de las empresas. El artículo está estructurado de la siguiente manera: en primer lugar, se amplían los conceptos de 'multilatinas' y 'reportes corporativos'; a continuación, se explica el desarrollo metodológico; luego, se presenta el análisis de los resultados, y finalmente se concluye con algunas consideraciones finales. 


\section{Conceptualización}

\section{Multilatinas}

En los últimos años han cobrado mayor importancia ciertas empresas dada su influencia en mercados latinoamericanos, no solo por la participación en estos y sus relaciones de competencia, sino también por el músculo financiero, así como el tejido social y ambiental que involucran. Se entiende por compañías latinas a aquellas del continente americano que resultaron de la colonización que se dio por parte de España, Portugal y Francia (Cuervo-Cazurra, 2010).

Aunque el uso del término de 'multilatinas' sea relativamente reciente (década del 90 en adelante), es un fenómeno con tradición. Para el profesor Cuervo-Cazurra, una multilatina es aquella colonizada por latinos pero que además "tienen operaciones de valor añadido fuera de su país de origen" (2010, p. 16). La razón por la que se considera un tema de actualidad y en tendencia es el liderazgo y tamaño de las empresas multilatinas de mejor desempeño financiero en la actualidad (Castro et ál., 2012).

Las primeras multinacionales latinoamericanas comenzaron procesos de exportación desde finales del siglo xx (Castro et ál., 2012); sin embargo, Latinoamérica muestra su desarrollo empresarial de manera más pronunciada en los últimos 50 años, y puede ser descrito en 3 fases:

1. 1970-1990. En esta fase, las empresas comenzaron a hacerse maduras en su negocio desenvolviéndose en sus mercados protegidos (Castro et ál., 2012) a través de la explotación de sus ventajas comparativas - recursos naturales, costo de la mano de obra, apoyo gubernamental, posición geográfica, entre otras- (Cuervo-Cazurra, 2010).

2. 1990-2002. Fase marcada por el consenso de Washington. Producto de lo que se conoció como década perdida, se tuvieron que llevar a cabo reformas promercado que recogieran las sugerencias realizadas por el Fondo Monetario Internacional (FMI) para mitigar la caída del ingreso 
per cápita, la alta inflación, el crecimiento de la deuda externa, caída del salario real, entre otros (Sarmiento, E. 1990). Así, se implementaron diferentes tipos de cambio competitivos, liberalización de comercio internacional e inversión extranjera directa, privatización; entre otras medidas (Cuervo-Cazurra, 2007).

3. 2002 y siguientes. Producto del aprovechamiento de los tratados de libre comercio convenidos entre países de la región, se incrementó la presencia de compañías extranjeras en los mercados locales, lo que forzó a reestructurar las compañías para dinamizar sus procesos y buscar oportunidades en mercados foráneos (Castro et ál., 2012).

\section{Reportes corporativos}

La dinámica de reportar información no financiera, que acompaña la información financiera, es una tendencia creciente que ha sido adoptada por las empresas desde hace más o menos tres décadas, cuando el mundo entero sintió a todo nivel los efectos adversos de la falta de información y, a la vez, de su falta de calificación (Gómez-Villegas y Quintanilla, 2012).

Ahora bien, dicha tendencia de revelación de información fue potenciada porque las empresas de manera indirecta podían sentir un beneficio mayor al costo de preparación y presentación de información, entendiendo por beneficios la disminución de los costos de capital, de costos políticos y legales, para contribuir al aumento de confianza de los inversionistas. Esto, al final, conduce a una mejora de la imagen que proyecta la compañía (Correa-García et ál., 2016).

Se entiende, entonces, que la información revelada por las empresas en sus reportes debe tener un volumen adecuado en cuanto a su extensión, pero que contenga información de calidad, clara y útil, para que contribuya a la toma de decisiones de sus usuarios (Correa-García et ál., 2016). De esta manera, se entiende que la falta de información no tan positiva dentro de las revelaciones se relaciona con malas noticias (Larrán-Jorge y García-Mecca, 2004). 
Actores sociales como el Príncipe de Gales, e instituciones como la firma KCGP, las Naciones Unidas, la Junta de Normas Internacionales de Contabilidad (IASB), la Iniciativa de Reporte Global (GRI), el International Integrated Reporting Council (IIRC), entre otros, se encaminaron en la definición de un marco de referencia de revelación integral (Correa-García et ál., 2016). Para el presente artículo, se consideran los lineamientos de revelación la GRI, que es una organización internacional independiente pionera en el reporte sostenible y emite guías para la revelación de dicha información y medida por indicadores definidos, actualmente bajo la guía G4, y del IIRC, coalición internacional que definen el concepto de Reporte Integrado. Estas instituciones sirven como guía pues reúnen una visión más completa de lo que se concibe como pensamiento integrado.

El concepto de 'reporte integrado', según el IIRC, es "una comunicación acerca de cómo la estrategia de una organización, su gobierno corporativo, desempeño y perspectivas, en el contexto de su entorno externo, la conducen a crear valor en el corto, mediano y largo plazo" (2013, p. 8). Dicho concepto puede ser complementado por el ofrecido por Correa-García et ál. (2018, p. 6), según el cual el reporte integrado "incluye información financiera y el reporte de sostenibilidad, además es preparado bajo los requerimientos del IIRC y los grupos empresariales hacen una declaración explícita de eso". Los autores, además, distinguen los reportes combinados, los cuales se componen del reporte de sostenibilidad (información respectiva a la responsabilidad social corporativa $-\mathrm{RSC}-$, realizados generalmente bajo estándares de la GRI) y la información financiera (tabla 1).

Tabla 1. Tipos de reportes y emisor de lineamientos

\begin{tabular}{|l|c|c|c|}
\cline { 2 - 4 } \multicolumn{1}{c|}{} & \multicolumn{3}{c|}{ Organismo emisor } \\
\hline Tipo de reporte & IASB & GRI & IIRC \\
\hline Reporte anual & $\checkmark$ & $x$ & $x$ \\
\hline Reporte de sostenibilidad & $x$ & $\checkmark$ & $x$ \\
\hline Reporte combinado & $\checkmark$ & $\checkmark$ & $x$ \\
\hline Reporte integrado & $\checkmark$ & $\checkmark$ & $\checkmark$ \\
\hline
\end{tabular}

Fuente: elaboración propia. 


\section{Desarrollo metodológico}

Para caracterizar los reportes corporativos emitidos por las multilatinas colombianas, se consideró el periodo de 2016 a 2018 como espacio muestral. Se partió del fortalecimiento en prácticas de reporte en Colombia y evoluciones importantes, como la introducción de los 17 Objetivos de Desarrollo Sostenible (oDs) en el 2015, cuando se migró de los 8 Objetivos Del Milenio (oDM), Y el afianzamiento y difusión del uso de la guía G4 de la GRI por parte de las empresas.

Asimismo, se tomó como fuente la publicación realizada por la revista América Economía en la que se recopilan las 100 mejores multilatinas de cada año (América Economía, 2017; 2018; 2019). De allí, se seleccionaron las multinacionales colombianas, con el fin de contar con un muestreo de carácter no probabilístico de muestra intencionada con un número de 10 empresas por año ${ }^{3}$.

Como unidad de análisis fueron tomados los reportes corporativos que, consideramos, presentan un nivel mayor de información integrada (tabla 1) por año. Los seleccionados fueron evaluados bajo una metodología de análisis de contenido con el fin de analizar su proceso de comunicación y la calidad de su contenido (Fernández, 2002). Las categorías de análisis fueron seleccionadas partiendo de la guía G4 de 2013 del GRI, y se consideraron las dimensiones del reporte que presentan información material por tema. Como variables e indicadores, se obtuvieron los que se muestran en la tabla 2.

3 Para el 2017 hay 11 empresas listadas; sin embargo, una no cuenta con dicha información publicada. 
Tabla 2. Categorías de análisis e indicadores de la matriz

\begin{tabular}{|c|c|c|}
\hline Variable & Descripción & Indicadores \\
\hline \multirow{4}{*}{ 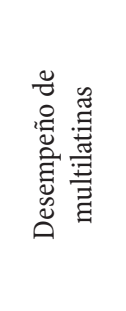 } & \multirow{4}{*}{$\begin{array}{l}\text { Con el propósito de establecer cuáles son las } 100 \\
\text { multilatinas más destacadas de cada año, la revista } \\
\text { América Economía recopila información acerca de las } \\
\text { ventas, número de empleados, presencia geográfica } \\
\text { y otros análisis especiales en los que suma } 30 \\
\text { indicadores, de los cuales se tomaron } 7 \text { considerados } \\
\text { relevantes para entender la evolución de la empresa } \\
\text { como multilatina en los últimos } 3 \text { años. } \\
\end{array}$} & Presencia países \\
\hline & & Empleados totales \\
\hline & & $\begin{array}{l}\% \text { empleados en el } \\
\text { exterior }\end{array}$ \\
\hline & & $\begin{array}{l}\% \text { variación de } \\
\text { ventas }\end{array}$ \\
\hline \multirow{3}{*}{ 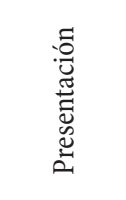 } & \multirow{3}{*}{$\begin{array}{l}\text { La estructura del reporte es fundamental para que } \\
\text { la información contenida en él resulte clara y útil } \\
\text { para los lectores. Se espera que las organizaciones se } \\
\text { hayan inclinado por la integración de la información, } \\
\text { aunque emitan varios. }\end{array}$} & Nombre \\
\hline & & Concepto reporte \\
\hline & & $\mathrm{N} .^{\circ}$ de páginas \\
\hline \multirow{2}{*}{ 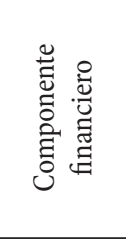 } & \multirow{2}{*}{$\begin{array}{l}\text { No se debe olvidar que la información financiera es } \\
\text { muy relevante dentro del proceso de comunicación } \\
\text { de información, en tanto comunica la situación } \\
\text { financiera y económica de las organizaciones, por } \\
\text { medio de los estados financieros y demás análisis de } \\
\text { indicadores. }\end{array}$} & $\mathrm{N} .^{\circ}$ de páginas \\
\hline & & Firma auditora \\
\hline \multirow{3}{*}{ 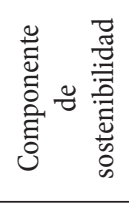 } & \multirow{3}{*}{$\begin{array}{l}\text { Para este caso comprende las perspectivas sociales y } \\
\text { ambientales, que, para Correa-García et ál. (2016), } \\
\text { son "infaltables". Este componente representa un } \\
\text { reto para las organizaciones en cuestión de reportar } \\
\text { información real (tanto positiva como negativa). }\end{array}$} & Adherencia GRI \\
\hline & & $\%$ oDs \\
\hline & & $\begin{array}{l}\text { Verificación } \\
\text { externa }\end{array}$ \\
\hline \multirow{6}{*}{ 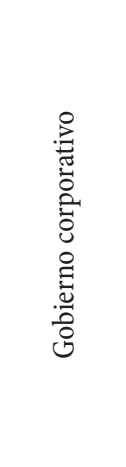 } & \multirow{6}{*}{$\begin{array}{l}\text { Es importante incluir el gobierno corporativo pues } \\
\text { este no solo influye en la toma de decisiones de la } \\
\text { compañía, sino también en la posible información } \\
\text { por revelar. Entender cómo está compuesto y } \\
\text { la estructura societaria de la compañía ayuda a } \\
\text { comprenderla de una manera integral. }\end{array}$} & $\begin{array}{l}\text { Miembros de la } \\
\text { junta directiva (JD) }\end{array}$ \\
\hline & & Independencia JD \\
\hline & & Mujeres JD \\
\hline & & $\begin{array}{l}\text { Accionista } \\
\text { mayoritario }\end{array}$ \\
\hline & & $\begin{array}{l}\% \text { accionista } \\
\text { mayoritario }\end{array}$ \\
\hline & & $\begin{array}{l}\text { País del accionista } \\
\text { mayoritario }\end{array}$ \\
\hline
\end{tabular}

Fuente: elaboración propia. 


\section{Análisis de resultados}

A continuación, se presentan algunas apreciaciones como resultados para cada categoría de análisis.

\section{Desempeño de las multilatinas}

Como se muestra en la figura 1 , se encontró que solo el $20 \%$ de las empresas logró mantenerse dentro del primer cuartil de las 100 mejores; $30 \%$, en el segundo —entre la empresa número 26 y el 50 - y el $50 \%$, dentro del tercer cuartil durante los 3 años analizados. Se destaca que la única empresa que logró mejorar su posición en el escalafón cada año fue Colombina, aunque permaneció siempre entre la empresa número 51 y 75 (74-72-61 respectivamente).

\section{Figura 1. Empresas por cuartil}

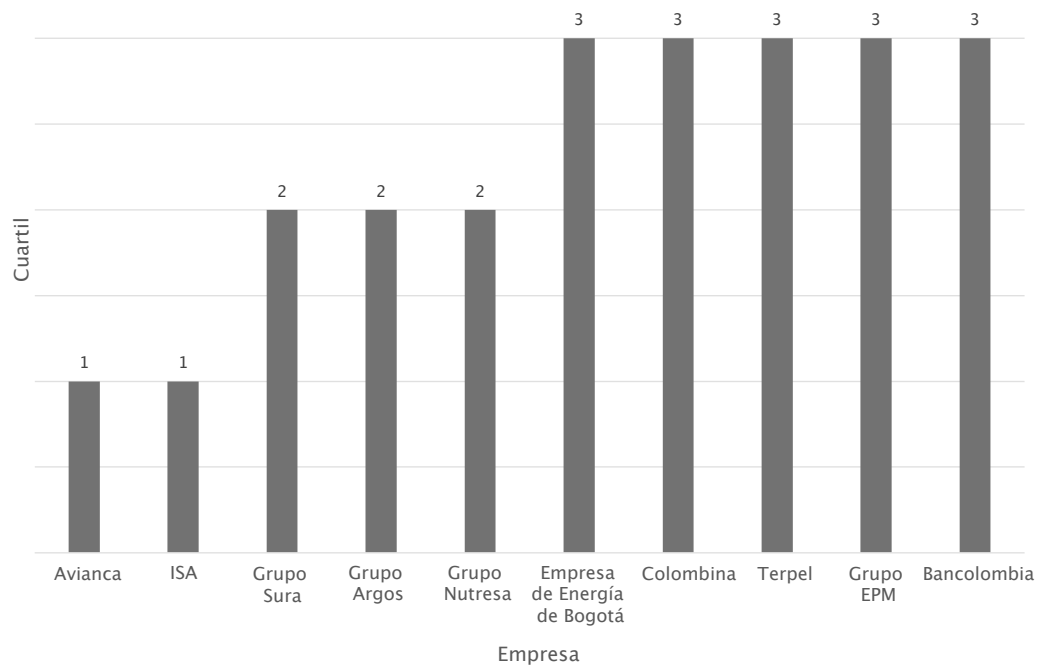

Fuente: elaboración propia. 
Los procesos de crecimiento propios de cada multilatina son tan específicos que, según Castro et ál. (2012), es difícil reconocer patrones de internacionalización y, en este caso, patrones de evolución de los reportes corporativos. Lo mencionado se refleja en que, a pesar de que el $50 \%$ de las empresas aumentó el número de países donde hacen presencia, cuatro de ellas aumentaron su cantidad de empleados en el exterior, pero en solo dos crecieron sus ventas; por otro lado, las empresas que salieron de países (30\%) sí reflejaron la disminución de sus empleados, pero con un extraño crecimiento en el nivel de ventas en el exterior. Para completar las irregularidades, de las dos empresas que mantuvieron estable el número de países, una aumentó su nivel de ventas, pero su número de empleados disminuyó y en la otra sucedió todo lo contrario.

\section{Presentación}

Siguiendo las características establecidas para cada concepto de reporte, según el nivel de integración de información que presenta en su contenido y el cumplimiento que da a lineamientos de diferentes emisores, se puede evidenciar que el $60 \%$ de los reportes son bien nombrados por las empresas (cuatro empresas denominan sus reportes "integrados", una los denomina "de sostenibilidad" y otra hace la distinción entre "anuales" y "de sostenibilidad”). En efecto, el nombre con el que publican su información corresponde a lo desarrollado en su contenido, lo cual es considerado como un acierto porque desde el inicio le ofrece al usuario el contenido que se espera desde el título del reporte (figura 2).

En cuanto al número de páginas de los reportes, este indicador, para efectos del artículo, es medido con el propósito de identificar la variación de este para dicho periodo. Sin embargo, cada reporte debe ser analizado de manera individual, pues si un año a otro se presentan más páginas, no quiere decir en todos los casos que se esté comunicando más información. Por ejemplo, se debe reconocer la concisión con la cual se abordan los temas materiales del informe sin dejar de lado la extensión necesaria con la que se debe introducir un tópico, que en cada caso es relativo a cada compañía. 
Figura 2. Nombre del reporte en relación con su contenido

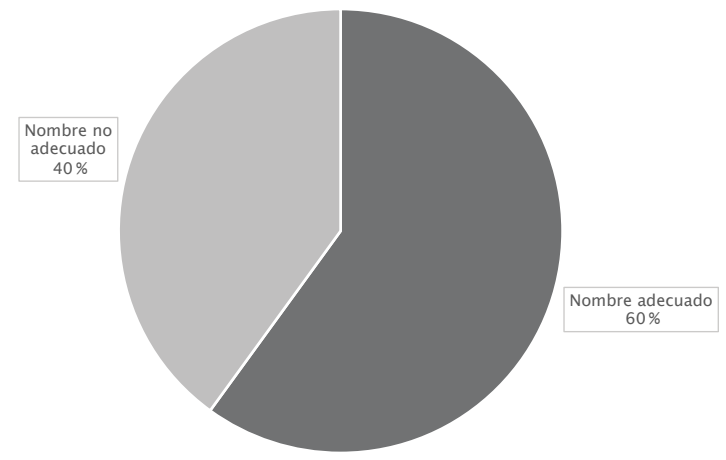

Fuente: elaboración propia.

Dicho esto, se pudo reconocer que $40 \%$ de las empresas aumentaron la cantidad de páginas que integró más información para cada año, $30 \%$ disminuyó la cantidad cada año y el 30 \% tuvo una evolución irregular en el periodo. El Grupo Sura pasó de publicar informes de más de 650 páginas en 2016 y 2017 a publicar uno de 154 en el último año; además, el Grupo EPM publicó reportes de más de mil páginas cada año (figura 3).

Figura 3. Comportamiento del número de páginas

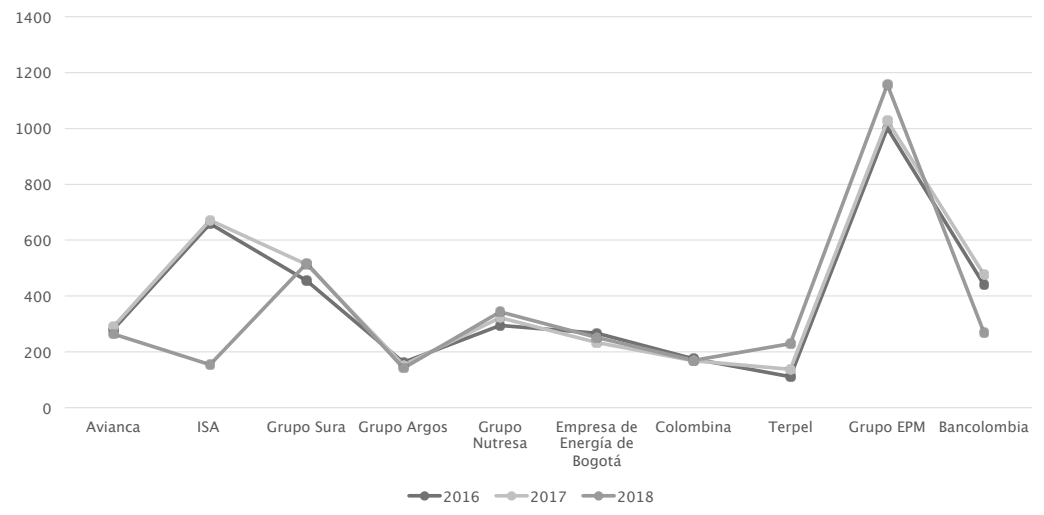

Fuente: elaboración propia. 


\section{Componente financiero}

De las 9 empresas que reportaron los estados financieros dentro de su informe (Terpel no presentó dicha información), en promedio se destina aproximadamente el $34 \%$ de las páginas totales a este tipo de contenido; sin embargo, se puede evidenciar que varía significativamente entre las empresas (figura 4).

Figura 4. Porcentaje de contenido promedio de información financiera de los reportes

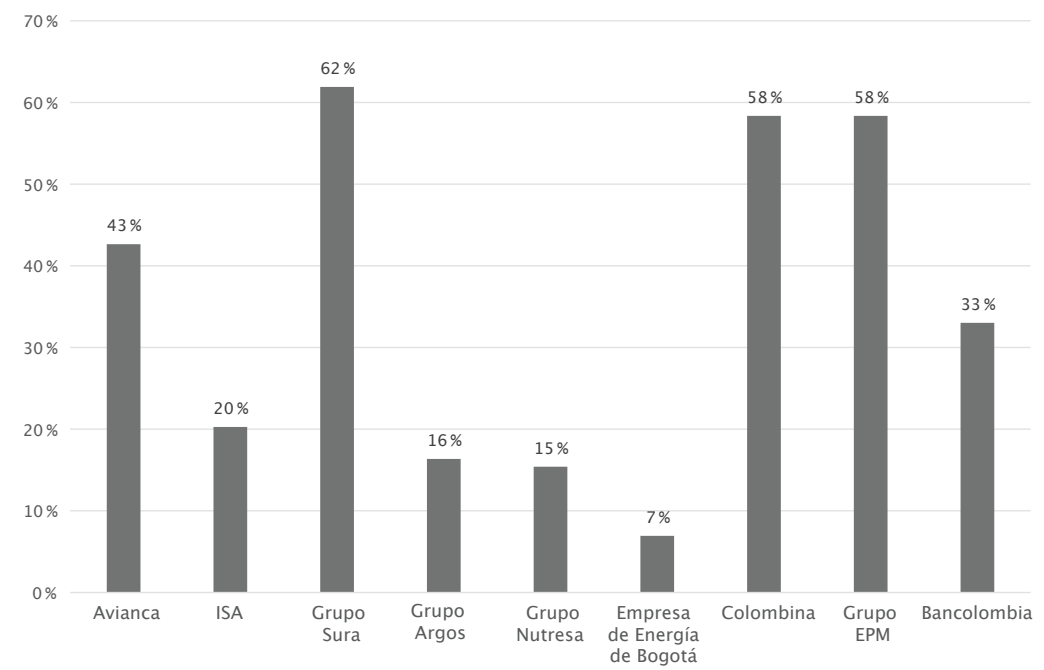

Fuente: elaboración propia.

El 46.67 \% de los informes presentan dualidad de revisión dentro del componente financiero y el de sostenibilidad, al ser auditados y verificados por la misma compañía, mientras que el $30 \%$ de los informes son auditados y verificados por compañías diferentes (el restante $23.33 \%$ corresponde a casos en los que alguno de los dos o ambos no son sometidos a revisión por terceros o no fueron presentados). 


\section{Componente de sostenibilidad}

Del total de reportes analizados, aproximadamente el $73 \%$ fue realizado con un nivel de adherencia esencial (core) según la guía G4 de 2013 de la GRI. Esto quiere decir que cumplieron con reportar información sobre algunos de sus indicadores, mientras que solo el $20 \%$ se adhirió por completo a los indicadores para estar en un nivel exhaustivo (comprehensive) y aproximadamente el 6\% no desarrolló sus informes bajo la guía de la GRI.

Figura 5. Adherencia a las guías de la GRI

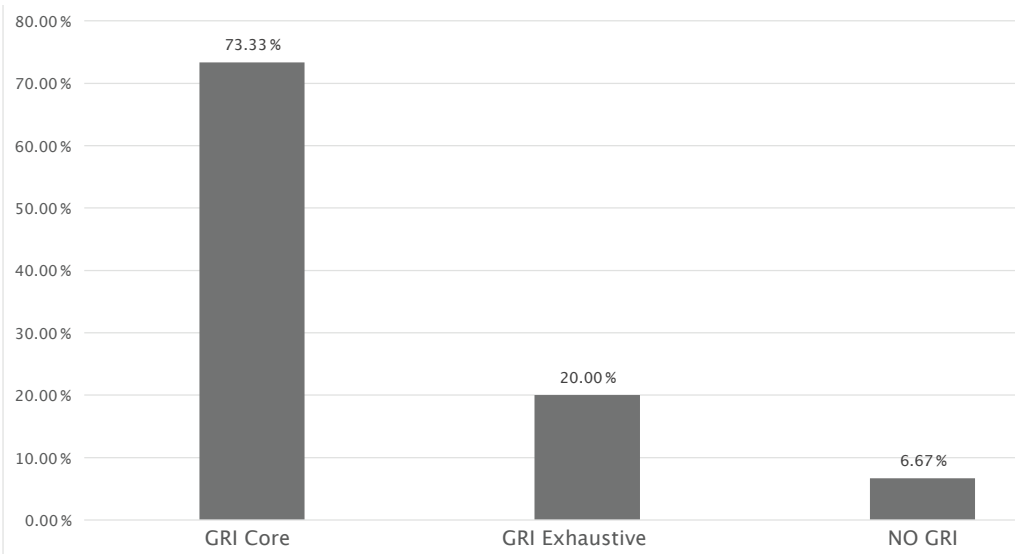

Fuente: elaboración propia.

En cuanto a los Objetivos de Desarrollo Sostenible, este grupo de empresas tuvo como objetivos más priorizados durante todo el periodo al número 8 ("trabajo decente y crecimiento económico") y el 9 ("innovación e infraestructura"), lo que en primera instancia indica que las empresas están enfocadas fundamentalmente en contribuir a la sociedad por medio de sus procesos de mejora continua. En segundo lugar, las empresas informan que enfocan su esfuerzo en contribuir con el oDs 4 ("educación de calidad") directamente con población infantil, y el 13 ("acción por el clima"), que relaciona a la organización con obras en beneficio de la preservación del medio ambiente. Parten sobre la base de que los 17 fueron planteados para 
que las empresas puedan comprometerse en el marco de la agenda 2030 a velar por el cumplimiento del mayor número de objetivos y mantener dichos procesos en el tiempo, para contribuir al desarrollos sostenible (obrar bien en el presente para garantizar un futuro mejor).

En cuanto a la dinámica propia del cumplimiento de los oDs en estas empresas, desde que se implementó en cada análisis de materialidad la relación de la empresa y su obra asociada a ellos, se pudo reconocer que en el $60 \%$ de las empresas el número de objetivos creció o después de crecer se mantuvo. En el $20 \%$ estuvo estático durante el periodo analizado y otro 20 \% disminuyó su relación. Este resultado no responde solo a una causa, por lo que debe ser estudiado cada caso de manera particular.

Cabe resaltar que tanto la verificación externa del contenido relacionado con temáticas de sostenibilidad como la auditoría del componente financiero fue realizada por las Cuatro Grandes (Big Four).

Tabla 3. Firmas de auditoría financiera y verificación externa según número de reportes

\begin{tabular}{|l|c|c|c|c|}
\hline \multicolumn{1}{|c|}{ Empresa } & Financiera & $\begin{array}{c}\text { \% de } \\
\text { participación }\end{array}$ & Verificación & $\begin{array}{c}\text { \% de } \\
\text { participación }\end{array}$ \\
\hline Deloitte & 13 & 43.3 & 17 & 56.6 \\
\hline Ernst \& Young & 7 & 23.3 & 3 & 10 \\
\hline KPMG & 4 & 13.3 & 6 & 20 \\
\hline $\begin{array}{l}\text { Price Waterhouse } \\
\text { Coopers }\end{array}$ & 4 & 13.3 & 0 & 0 \\
\hline $\begin{array}{l}\text { No enunciado en el } \\
\text { reporte }\end{array}$ & 2 & 6.6 & 4 & 13.3 \\
\hline Total de reportes & 30 & 100 & 30 & 100 \\
\hline
\end{tabular}

Fuente: elaboración propia. 


\section{Gobierno corporativo}

Entendiendo al gobierno corporativo como máxima autoridad en toma de decisiones, se debe analizar su junta directiva y la junta de socios (en este caso la composición de capital) de modo que se pueda dilucidar a grandes rasgos cómo son orientadas estas organizaciones hacia la generación de valor. Para el 67 \% de las empresas con información completa de su junta directiva (pues Colombina no publica dicha información en sus reportes), el nivel de independencia es superior al $50 \%$, mientras que para el restante $33 \%$ su nivel de independencia es inferior al $50 \%$. Dichos datos, de entrada, proyectan el comportamiento de estas empresas colombianas en el cumplimiento de lo propuesto por la teoría de la agencia.

Figura 6. Nivel de independencia de la junta directiva

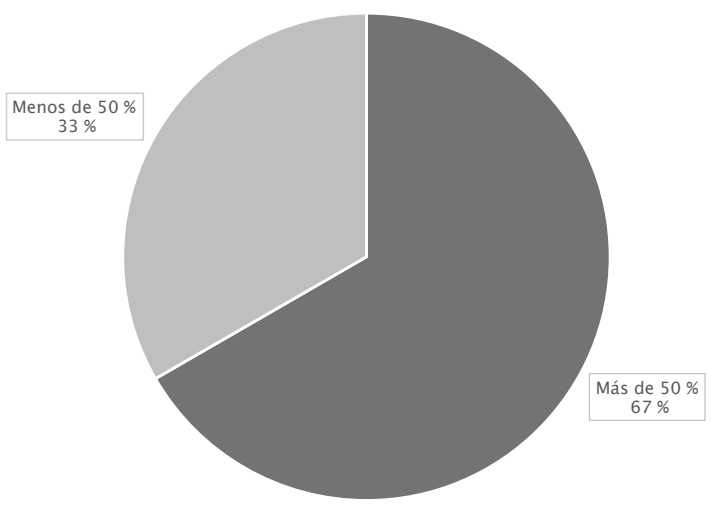

Fuente: elaboración propia.

En los últimos 3 años, 7 empresas incluyeron mujeres en su junta directiva, y de ellas el $28.57 \%$ tuvo al menos una mujer durante un año; el 14.29 $\%$, durante dos años, y el $57.14 \%$ tuvo al menos una mujer durante 3 años. Adicionalmente, de las 8 empresas que comunican información acerca de su composición accionaria, 7 son controladas por compañías colombianas y 1 por una compañía chilena, lo que puede indicar en primera instancia que 
la dinámica de los reportes de estas compañías corresponde a decisiones propias del contexto local.

\section{Consideraciones finales}

Producto de los análisis realizados, no se puede afirmar que exista una tendencia marcada entre el 2016 y el 2018 por las empresas multilatinas colombianas a presentar información por medio de reportes integrados 0 , de manera similar, por medio de reportes que presenten una información combinada para dotar de multidimensionalidad a la información publicada, de manera que se dirijan al mayor número de grupos de interés o stakeholders posibles.

Con la investigación no se aborda el cumplimiento de los compromisos adquiridos por las empresas con la sociedad como resultados de acciones relacionados con RSC y que fueron plasmados en sus publicaciones. Además, aunque se considere que la rendición de cuentas está dotada implícitamente de transparencia, no siempre son conceptos complementarios (GómezVillegas y Quintanilla, 2012).

La ausencia de patrones comunes en la dinámica de reportar información es respaldada en que, a pesar de buscar la unificación de conceptos por medio de la instrumentalización, se siguen respondiendo a entornos de Rsc propios de cada empresa, que de perder el carácter de voluntariedad iría contra uno de los principios básicos (Gómez-Villegas y Quintanilla, 2012). Asimismo, las prácticas de reporte corporativo son principalmente asumidas por grandes empresas y, más allá de su motivación particular, se debe partir de la buena fe y la filantropía, características de la RSC.

En ocasiones, no se puede reconocer dinámica de integración de información en los reportes corporativos dados los cambios en el contenido y su publicación, lo cual no quiere decir que la empresa no siguió cumpliendo con el proceso que inició en periodos anteriores, pero tampoco lo hace evidente. Así, es necesario un análisis exhaustivo de cada reporte, pues 
pueden existir muchas más razones que no permitan identificar dicha dinámica, sin descartar la idea de que las empresas simplemente no están enfocadas en una práctica de reporte corporativo con miras a la integración multidimensional de contenidos.

Este artículo presenta un avance en la caracterización de los reportes presentados por las multilatinas, que, si bien ha sido un tema explorado, no se hacía una relación entre estas importantes empresas para América Latina y Colombia con los reportes corporativos. Es menester seguir trabajando en estos temas que representan para la profesión contable nuevos retos en cuanto a su participación como actores que juegan un papel central como dadores de fe pública en la información que las empresas comunican.

Se extiende la invitación a que se siga investigando de manera interdisciplinar y enfocada tanto los fenómenos de internacionalización y crecimiento de empresas como la adopción de marcos para la preparación de reportes corporativos y la reciente migración de Objetivos del Milenio a Objetivos de Desarrollo Sostenible, para que seguir aclarando las motivaciones e incidencias de reportar información de manera voluntaria.

\section{Referencias}

Asociación Española de Contabilidad y Administración de Empresas. (2011). Hacia la información integrada: la comunicación de valor en el siglo XXI. Consultado en http://aeca.es/old/comisiones/rsc/iirc_traduccion_espanol. pdf el 10/02/2020.

América Economía. (2017). Ranking Multilatinas 2016. Consultado en https:// rankings.americaeconomia.com/2016/multilatinas/ranking

América Economía. (2018). Ranking Multilatinas 2017.

América Economía. (2019). Ranking Multilatinas 2018.

Casanova, L. y Dumas, A. (2010). Corporate Social Responsibility and Latin American Multinationals: Is poverty a business issue? Universia Business Review, (32), 132-145. Consultado en http://www.redalyc.org/articulo. oa? $\mathrm{id}=43312280008$ 
Castro, J., Castro J. y Jaller, I. (2012). Internationalization Patterns of Multilatinas. Ad-minister, (21), 33-54. Consultado en http://publicaciones.eafit.edu.co/ index.php/administer/article/view/1726

Correa-García J., Hernández-Espinal, M., Vásquez-Arango, L. y Soto-Restrepo, Y. (2016). Reportes integrados y generación de valor en empresas colombianas incluidas en el índice de sostenibilidad Dow Jones. Cuadernos de Contabilidad, 17(43),73-108. Dor: http://dx.doi.org/10.11144/Javeriana.cc17-43

Correa-García, J-A., García-Benau, M-A., García-Meca, E. (2018). CSR Communication Strategies of Colombian Business Groups: An Analysis of Corporate Reports. Sustainability, 10, 1602. DoI: https://doi.org/10.3390/su10051602

Cuervo-Cazurra, A. (2007). Liberalización económica y Multilatinas. Globalization, competitiveness and gobernavility, 1(1), 66-87. Dor: https://doi.org/10.3232/ GCG.2007.V1.N1.03

Cuervo-Cazurra, A. (2010). Multilatinas. Universia Business Review, (25), 14-33. Consultado en https://dialnet.unirioja.es/servlet/articulo? codigo=3143803

Fernández, F. (2002). El análisis de contenido como ayuda metodológica para la investigación. Revista de Ciencias Sociales, 2(96), 34-53. Consultado en https:// www.redalyc.org/pdf/153/15309604

Gómez-Villegas, M. y Quintanilla, D. (2012). Los informes de responsabilidad social empresarial: su evolución y tendencias en el contexto internacional y colombiano. Cuadernos de Contabilidad, 13(32), 121-158. Consultado en https://revistas.javeriana.edu.co/index.php/cuacont/article/view/3894

International Integrated Reporting Council. (2013). El marco Internacional IR. Consultado en http://integratedreporting.org/wp-content/uploads/2015/03/1312-08-THE-INTERNATIONAL-IR-FRAMEWORK-SPANISH-1.pdf.

Larrán-Jorge, M., y García-Meca, E. (2004). La relevancia de la información no financiera en la estrategia empresarial de divulgación voluntaria: percepciones empresa-analista sobre su utilidad. Revista Valenciana de Economía y Hacienda, 12, 127-148. Consultado en https://dialnet.unirioja.es/servlet/ articulo? codigo $=1429785$ 